\title{
Changes in British men's use of family planning services between 1991 and 2000: secondary analysis of a national survey
}

\author{
Stephen C Pearson, Paul Clarke
}

\begin{abstract}
Background and methodology Although men are identified as a priority group for sexual health initiatives, limitations in other data sources mean little is known about their use of general practitioners (GPs) or family planning clinics (FPCs) for family planning services. This paper provides a unique profile of which men have increased their use of GPs or FPCs for family planning services over the 1990s. Data were taken from the repeated crosssectional British Omnibus Survey, 1991-2000. In total, 16470 men aged 16-49 years were asked which health service they had used for family planning purposes within the last 5 years.
\end{abstract}

Results Although around two-thirds of men cite general practice as their preferred source for professional advice on contraception, in 2000 only $12.5 \%(95 \% \mathrm{Cl} 11-14)$ had used this source for family planning purposes in the past
5 years. Between 1991 and 2000, the greatest growth was in use of FPCs, particularly among men aged 16-24 years, of single or cohabiting marital status, or of professional or unskilled social class. The greatest growth in use of GPs relative to use in 1991 was among men aged 35-44 years and those in the skilled non-manual and skilled manual social classes.

Discussion and conclusions As the 1990s progressed, an increasing percentage of men attended GPs and FPCs for family planning purposes. Differential rates of growth by age group, marital status and social class have occurred, but levels of use are still much lower than for women.

Keywords data analysis, family planning clinics, family planning service provision, general practice, men

J Fam Plann Reprod Health Care 2007; 33(3): 183-187

(Accepted 22 August 2006)

\section{Introduction}

Due to the comparatively low number of male clients, and limitations of routinely collected data, little is known about the characteristics of men using family planning services in Britain. ${ }^{1}$ The emergence of HIV in the mid-1980s and the continuing high rate of teenage conceptions in Britain ${ }^{2}$ have focused attention on the sexual health-seeking behaviour of men. The 1992 Health of the Nation initiative in England set an (unachieved) target to halve the conception rate for women aged 13-15 years by $2000 .^{3}$ More recent national policy documents on teenage pregnancy 4 and sexual health and HIV $^{5-7}$ address the range, type and appropriateness of sexual health services in Britain. These documents highlight the need to address the sexual health needs of men - particularly gay, bisexual and young men - to improve sexual health. Teenage mothers are more likely to come from a lower socioeconomic background, ${ }^{8}$ indicating the need to target men from poorer and socially excluded backgrounds.

Routinely collected data from family planning clinics (FPCs), Brook Advisory Centres and genitourinary medicine clinics suggest increasing male attendance over the 1990s. ${ }^{1}$ However, these datasets rarely include the demographic or socioeconomic characteristics of male clients with which to identify use among key groups. Although the number of male clients of FPCs increased $157 \%$ between $1990 / 1991$ and $1999 / 2000,{ }^{1}$ the ages of these clients were not recorded (unlike for female clients).

Nuffield Centre for International Health and Development, University of Leeds, Leeds, UK

Stephen C Pearson, PhD, Senior Research Fellow

Department of Epidemiology and Population Health, London School of Hygiene and Tropical Medicine, London, UK Paul Clarke, PhD, Senior Lecturer in Statistics

Correspondence to: Dr Stephen C Pearson, Nuffield Centre for International Health and Development, University of Leeds,

71-75 Clarendon Road, Leeds LS2 9PL, UK.

E-mail: S.C.Pearson@leeds.ac.uk

\section{Key message points}

- Between 1991 and 2000, the percentages of men attending family planning clinics (FPCs) and general practitioners (GPs) for family planning purposes increased.

- Groups with the highest rates of growth in use of FPCs are: men aged 16-24 years, of single and cohabiting marital status; and men from the professional and unskilled social classes.

- Groups with the highest rates of growth in use of GPs are men aged 35-44 years and men from skilled nonmanual and skilled manual social classes.

Recent national estimates of men's use of general practitioners (GPs) for family planning purposes are unavailable.

The objectives of this paper are to address these failings by providing a unique profile of men reporting use of FPCs or GPs for family planning purposes in the period 1991-2000, and the changes in usage over this period, using data from a national repeated cross-sectional survey.

\section{Methods}

The study data come from the National Statistics Omnibus Survey, a nationally (Great Britain) representative survey in which each month a batch of around 2000 interviews are carried out with adults aged 16 years or over. 9 Different individuals are selected for interview each month, in contrast to a longitudinal panel design where selected individuals are re-interviewed over time. The survey uses a multistage sampling design in which a sample of 100 postcode sectors, stratified by region and sector characteristics, is selected each month from the Postcode Address File with probability proportional to size; then 30 addresses/delivery points are selected from these sectors; and one eligible person is interviewed per household. Due to the one person per household sampling design, statistical analyses must be weighted to correct for unequal selection probabilities.

Between January 1991 and November 2000, a module 


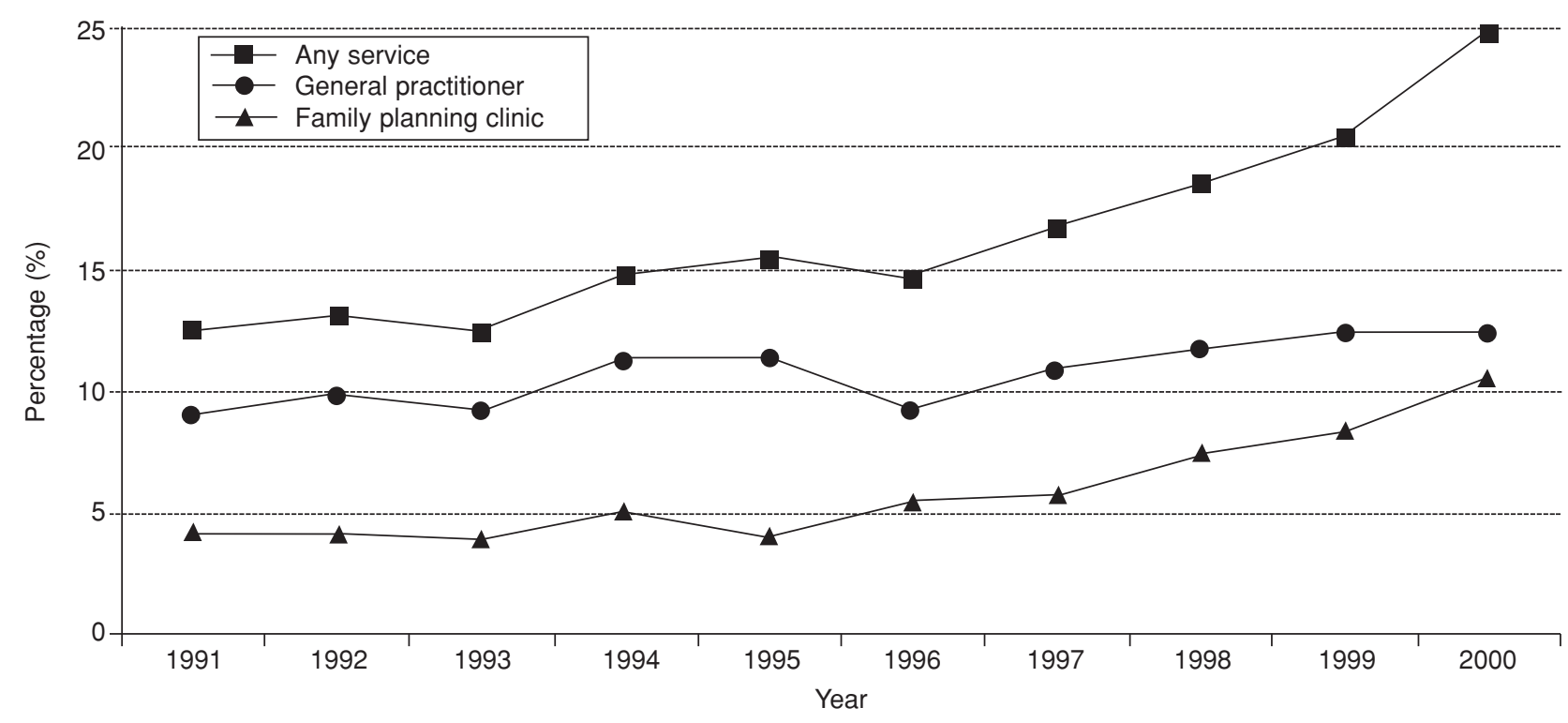

Figure 1 Percentages of men using any service, a general practitioner or a family planning clinic for family planning purposes in the last 5 years, 1991 to 2000 [NB. Refer to Table 1 for sample sizes and 95\% Cls for these estimates.]

of questions on contraception was asked in 37 of the monthly interview batches. These monthly data were weighted and aggregated to represent each calendar year. As few men aged 50 years or above report use of family planning services, only men aged 16-49 years are included in the analysis.

Family planning service use: Family planning service use measured whether the man had visited (a) a GP, (b) a FPC (defined in the questionnaire as including a Brook clinic or Marie Stopes clinic) or (c) any service [i.e. (a) or (b) or any other unnamed service] for family planning purposes within the last 5 years. In total, only 141/16 470 men $(0.9 \%)$ who had not attended either (a) or (b) had used another service, hence the focus on GPs and FPCs.

Age: Age was categorised into 5-yearly intervals.

Marital status: Four categories of marital status were used: married, cohabiting (including same-sex), single and widowed/divorced/separated.

Social class: The Registrar General's socioeconomic groups, based upon the 1990 standard occupation categories, were used to measure social class. This measure was chosen over other measures of social class or socioeconomic background for its completeness from 1991 to 2000. Men whose social class was classified as 'inadequately described', 'never worked' or 'armed forces' were excluded from the analysis.

\section{Statistics}

All statistical calculations were carried out in Stata (Version 8.0) (Stata Corp., College Station, TX) using the survey commands to produce estimates and their standard errors that are correctly adjusted for the sampling design. Time-series of service use are shown as the weighted percentages of men using the service in each year, together with the unweighted sample size. The trend in use over time is measured by relative change, which is defined as the per-year change in use from 1991 to 2000 expressed as a percentage of 1991 use. Relative change is estimated using logistic regression to minimise the influence of temporal and random fluctuations on estimated trend (see the Appendix for further details). Time-series within subgroups are summarised by percentage use in 1991, percentage use in 2000 , and relative change over the 10 year period (all estimates are weighted). The likelihood ratio test is used to test whether use in subgroup categories (e.g. age, marital status) in a given year is uniform with $p$ values given for the null hypothesis of equal use between subgroup categories. Finally, tests for positive trend are

Table 1 Percentages of men using any service, a general practitioner or a family planning clinic for family planning purposes in the last 5 years, 1991 to 2000

\begin{tabular}{|c|c|c|c|c|}
\hline \multirow[t]{2}{*}{ Year } & \multirow[t]{2}{*}{$n$} & \multicolumn{3}{|c|}{ Service used $[\%(95 \% \mathrm{Cl})]$} \\
\hline & & Any service & General practitioner & Family planning clinic \\
\hline 1991 & 1967 & $12.6(11-14)$ & $9.1 \quad(8-10)$ & $4.3 \quad(3-5)$ \\
\hline 1992 & 1457 & $13.2(11-15)$ & $9.9(8-11)$ & $(3-5)$ \\
\hline 1993 & 1040 & $12.5(10-15)$ & $9.3(8-11)$ & $4.0 \quad(3-5)$ \\
\hline 1994 & 1479 & $14.8(13-17)$ & $11.4(10-13)$ & $(4-6)$ \\
\hline 1995 & 1382 & $15.5(14-18)$ & $11.4(10-13)$ & $4.1 \quad(3-5)$ \\
\hline 1996 & 1823 & $14.7(13-16)$ & $9.3(8-11)$ & $5.5 \quad(4-7)$ \\
\hline 1997 & 2675 & $16.8(15-18)$ & $10.9(10-12)$ & $5.8 \quad(5-7)$ \\
\hline 1998 & 1584 & $18.6(17-21)$ & $11.8(10-13)$ & $7.5 \quad(6-9)$ \\
\hline 1999 & 1670 & $20.5(18-23)$ & $12.5(11-14)$ & $8.4(7-10)$ \\
\hline 2000 & 1393 & $24.8(22-27)$ & $12.5(11-14)$ & $10.6(9-12)$ \\
\hline Relative change ${ }^{a}$ & 16470 & $6.7 \quad(5-9)$ & $3.4 \quad(2-5)$ & $15.8(11-22)$ \\
\hline
\end{tabular}

aRelative change is the estimated per-year increase in use as a percentage of the expected use in 1991. For example, between 1991 and 2000 , the use of family planning clinics increased by $15.8 \%$ per year on use in 1991 . 
Table 2 Percentages of men using a general practitioner or a family planning clinic for family planning purposes in the last 5 years - by age group, 1991 and 2000

\begin{tabular}{|c|c|c|c|c|c|c|c|}
\hline \multirow[t]{2}{*}{ Service used } & \multicolumn{7}{|c|}{ Age group in years $[\%(95 \% \mathrm{Cl})]$} \\
\hline & $16-19$ & 20-24 & $25-29$ & $30-34$ & $35-39$ & $40-44$ & $45-49$ \\
\hline \multicolumn{8}{|l|}{ General practitioner } \\
\hline 1991 & $5.2 \quad(3-11)$ & $7.4 \quad(5-11)$ & $12.9(10-17)$ & $11.2(8-15)$ & $9.6(7-14)$ & $10.3(7-14)$ & $(2-9)$ \\
\hline 2000 & $5.5 \quad(2-12)$ & $9.9 \quad(5-17)$ & $13.4(9-20)$ & $15.3(11-20)$ & $14.9(11-20)$ & $15.7(11-21)$ & $9.6 \quad(6-15)$ \\
\hline Relative change & $3.8(-8-16)$ & $5.5(-2-13)$ & $1.8(-2-6)$ & $0.8(-2-4)$ & $3.2(0-7)$ & $6.7(0-13)$ & $4.3(-4-13)$ \\
\hline \multicolumn{8}{|l|}{ Family planning clinic } \\
\hline 1991 & $1.6 \quad(0-7)$ & $6.0(4-10)$ & $5.0 \quad(3-8)$ & $6.6(4-10)$ & $5.0 \quad(3-8)$ & $3.1 \quad(2-6)$ & $(1-5)$ \\
\hline 2000 & $17.1(11-26)$ & 20.7 (14-29) & $16.1(11-23)$ & $9.3(6-14)$ & $7.0 \quad(4-11)$ & 4.8 (3-9) & $4.6 \quad(2-9)$ \\
\hline Relative change & $48.0(1-95)$ & $35.7(15-56)$ & $20.1(6-34)$ & $8.8(1-17)$ & $4.6(-4-13)$ & $4.2(-5-14)$ & $7.5(-10-25)$ \\
\hline$n$ & 1249 & 1712 & 2595 & 2930 & 2935 & 2655 & 2394 \\
\hline
\end{tabular}

based on whether 0 lies in the $95 \%$ confidence interval (95\% CI) for relative change; this test is equivalent to a formal test of trend (see the Appendix).

\section{Results}

The response rate for the Omnibus Survey varies by month, but in $200010 \%$ of eligible addresses were noncontactable, and a further $28 \%$ refused. 10 Of those men aged 16-49 years who agreed to participate in the Survey, around $4 \%$ refused to complete the module of questions on contraception, resulting in a sample of 16470 men.

The time-series of family planning service use between 1991 and 2000 shows the percentage of men who have used family planning services has increased, with the greatest relative change in the use of FPCs (Figure 1). In 2000, the estimated percentages using FPCs and GPs were $10.6 \%$ (95\% CI 9-12) and $12.5 \%$ (95\% CI 11-15), respectively (Table 1). Between 1991 and 2000, the relative change for FPCs was $15.8 \%$ (95\% CI 11-22) compared to $3.4 \%$ (95\% CI 2-5) for GPs; both of these increases are statistically significant.

Analysis of use of services by age group (Table 2) shows that use of GPs was highest in 2000 among age groups $30-44$ years (likelihood ratio test $p<0.03$ ). Although there is a trend towards higher use of FPCs in 2000 by younger age groups, the difference is not statistically significant (likelihood ratio test $p=0.10$ ) and could be due to sampling variation. Between 1991 and 2000, the estimated relative change in GP use is significant only for ages 35-39 and 40-44 years. For FPC use, the relative change between 1991 and 2000 is statistically significant for the four younger age groups (16-34 years); there is no change in the older age groups (35-49 years), although the CIs are wide. Note that the percentage of 45-49-year-olds using GPs was 4.8\% (95\% CI 2-9) in 1991 and $9.6 \%$ (95\% CI 6-15) in 2000, but the estimated relative change of $4.3 \%(95 \% \mathrm{CI}-4-13)$ is nonsignificant. This is because the percentage use in 2000 represents a large time-series fluctuation that is not representative of the overall 10-year trend.

The summary of the time-series of service use by marital status (Table 3 ) shows the relative change in GP use was largest among the single group (14.4\%, 95\% CI 4-25). For use of FPCs, all but the widowed/divorced/separated group showed large relative change, with an estimated $26.7 \%$ (95\% CI 5-49) and 49.0\% (95\% CI 23-75) relative change among the cohabiting and single groups, respectively. In 2000, use of a GP was higher among the cohabiting and married groups, whereas use of FPCs was higher among cohabiting and single groups.

Significant change in FPC use occurred among all the social class groups, with the largest change among the professional and unskilled (manual) groups [58.0\% (95\% CI 0-121) and 56.0\% (95\% CI 0-138), respectively] (Table 4). More modest changes in GP use can be seen among the two skilled groups [9.5\% (95\% CI 0-19) and 4.9\% (95\% CI 1-9) for the skilled non-manual and skilled manual groups, respectively], but there was no significant change for the other groups.

\section{Discussion}

This analysis of data from the British Omnibus Survey confirms that male attendance at health services for family planning purposes has increased over the 1990s. The greatest increase has been in the use of FPCs. In 2000, percentage use of GPs was highest among men in the age groups 30-44 years, of married or cohabiting marital status, and men of intermediate and skilled manual social classes. Percentage use of FPCs in 2000 was highest among men in the age groups 16-29 years, of cohabiting or single marital status, and the manual social classes.

Table 3 Percentages of men using a general practitioner or a family planning clinic for family planning purposes in the last 5 years - by category of marital status, 1991 and 2000

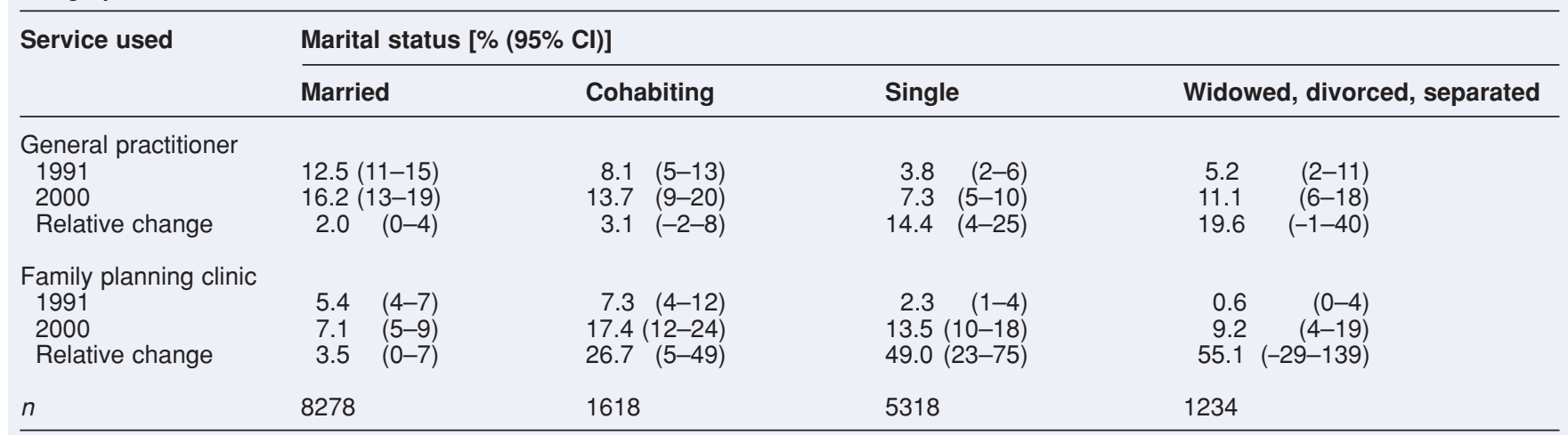


Table 4 Percentages of men using a general practitioner or a family planning clinic for family planning purposes in the last 5 years - by category of social class, 1991 and 2000

\begin{tabular}{|c|c|c|c|c|c|c|}
\hline \multirow[t]{2}{*}{ Service used } & \multicolumn{6}{|c|}{ Social class $[\%(95 \% \mathrm{Cl})]$} \\
\hline & Professional & Intermediate & $\begin{array}{l}\text { Skilled } \\
\text { non-manual }\end{array}$ & Skilled manual & Partly skilled & Unskilled \\
\hline \multicolumn{7}{|l|}{ General practitioner } \\
\hline 1991 & $3.5(1-13)$ & $12.4(9-16)$ & $4.6(2-10)$ & $10.2(8-13)$ & $6.7(4-12)$ & $7.0(3-16)$ \\
\hline 2000 & $9.0(5-16)$ & $14.9(12-19)$ & $11.9(8-18)$ & $12.6(9-17)$ & $12.3(8-18)$ & $9.2(4-20)$ \\
\hline Relative change & $5.3(-5-16)$ & $0.5(-2-3)$ & $9.5(0-19)$ & $4.9(1-9)$ & $1.8(-3-7)$ & $10.2(-4-25)$ \\
\hline \multicolumn{7}{|c|}{ Family planning clinic } \\
\hline 1991 & $0.9 \quad(0-6)$ & $4.8 \quad(3-8)$ & $3.1(1-7)$ & $3.9(3-6)$ & $6.2(3-11)$ & $2.0 \quad(0-8)$ \\
\hline 2000 & $10.1 \quad(6-18)$ & $7.5 \quad(5-11)$ & $9.6(6-16)$ & $10.4(7-14)$ & $12.6(8-19)$ & $6.4(5-19)$ \\
\hline Relative change & $58.0(0-121)$ & $8.7(0-17)$ & $11.9(0-27)$ & $19.1(8-31)$ & $10.4(0-24)$ & $56.0(0-138)$ \\
\hline$n$ & 1194 & 4348 & 1766 & 4632 & 2123 & 773 \\
\hline
\end{tabular}

Between 1991 and 2000, the highest growth relative to 1991 levels in use of GPs was among men of the widowed/divorced/separated marital status, and skilled non-manual and skilled manual social classes. Far higher rates of growth were seen in the use of FPCs; especially in men aged 16-24 years, the single and cohabiting marital statuses, and professional and unskilled social classes

To our knowledge, this analysis presents the first population estimates of the percentage of British adult men attending GPs and FPCs for family planning purposes, and the first estimates categorised by age group, marital status and social class. The analysis therefore provides information unavailable from routine client statistics, ${ }^{11}$ or previous reporting of the same topic from the Omnibus Survey ${ }^{10}$ or morbidity statistics from general practice. ${ }^{12}$

It is important to note some limitations with this study. Use of services for family planning purposes among men is an infrequent event; so even with a reference period of 5 years preceding the survey, some estimates have wide confidence intervals. In particular, the confidence intervals for relative change in social class groups are very wide here, reflecting the sensitivity of relative change to sampling variability when 1991 use is low. Larger sample sizes are necessary to estimate relative change with precision when 1991 use is low. However, both intervals here are informative in that they tell us the increases are statistically significant. Lastly, the unavailability of measures of contraceptive use, sexual activity, sexual orientation and alternative measures of socioeconomic status for 1991 to 2000 also weakens the analysis.

The uniqueness of this analysis means few comparisons can be made with other published sources. The overall increase in use of FPCs among men from 1991 to 2000 corresponds to the increase in the number of male clients recorded at FPCs for the same period. ${ }^{11}$ The larger relative change in use of FPCs by men aged 16-24 years corresponds to the increase in the number of male clients of this age group recorded at Brook Advisory Centres. ${ }^{13}$

The percentages of men in this analysis reporting use of a GP or FPC for family planning purposes are much lower than the percentages of women reported elsewhere. Women's responses from the 2000 Omnibus Survey, for example, suggest that around $50 \%$ of women used a GP, and $20 \%$ used a FPC, for family planning purposes in the last 5 years. ${ }^{10}$ Despite low utilisation rates among men, growth has occurred over the 1990s, perhaps reflecting changes in perceived need for services among men, and the accessibility and quality of services. The substantial growth in use of FPCs among men aged 16-24 years, and men from manual social classes is encouraging, given these groups were identified as targets for initiatives aiming to prevent sexual ill-health and teenage conceptions. ${ }^{5-7}$

There are four main reasons why men might attend a family planning service: for vasectomy services, male condoms, general advice or information on family planning, or to accompany a female partner. The few male-oriented methods of family planning available and the fact that GPs are unable to prescribe male condoms help explain men's low utilisation rates, and differential rates by age group and marital status. It is likely that many of the middle-aged, married and cohabiting men using GPs are seeking vasectomy services; analysis using the General Practice Research Database confirms incidence of male sterilisation is highest among the 30-39 years age group. ${ }^{14}$ Similarly, many of the young, cohabiting and single men using FPCs are likely to be seeking male condoms.

Although few men report use of a GP for family planning purposes, data recently released give one possible indication of unmet need in this group. In the National Survey of Sexual Attitudes and Lifestyles II 2000-2001, respondents were presented with a list of sexual health services and asked: "If all the different types of service were available in your area now and easy to get to, which one do you think you would prefer to use for professional advice on contraception?" General practice was the most preferred source: $52.6 \%$ of men aged 16-44 years chose a GP at their GP's surgery, and $14.9 \%$ chose a FPC at their GP's surgery. ${ }^{15}$ Despite this apparent preference for general practice, the present data suggest that only $12.5 \%$ of men in 2000 reported use of a GP for family planning purposes in the last 5 years. Is it that men using male condoms think they do not need professional advice, merely an accessible outlet for supply? Or would men be more likely to consider using general practice if male condoms were available combined with general sexual health and family planning services? Further research is needed to answer these questions, although condom provision schemes through general practice have had mixed success in attracting male clients. ${ }^{1}$

Continued monitoring and analysis of the Omnibus Survey data will reveal whether the trends identified in this article are being sustained, and the introduction of additional variables on contraception and sexual behaviour will allow a more refined identification of the men using general practice and FPCs for family planning purposes, and how health services can respond to these men's needs. 
Acknowledgements

The authors wish to thank Fiona Steele, James Newell and the three anonymous peer reviewers for their insightful comments on this study.

Statements on funding and competing interests

Funding None identified.

Competing interests None identified.

\section{References}

1 Pearson S. Men's use of sexual health services. J Fam Plann Reprod Health Care 2003; 29(4): 190-194.

2 Office for National Statistics. Report: Conceptions in England and Wales, 2003. Health Stat Q 2005; 26: 58-61.

3 Department of Health. The Health of the Nation: A Strategy for Health in England. London, UK: HMSO, 1992.

4 Social Exclusion Unit. Teenage Pregnancy. London, UK: The Stationery Office, 1999.

5 Department of Health. The National Strategy for Sexual Health and HIV. London, UK: Department of Health, 2001.

6 Scottish Executive. Respect and Responsibility: Strategy and Action Plan for Improving Sexual Health. Edinburgh, UK: Scottish Executive, 2005.

7 The National Assembly for Wales. A Strategic Framework for
Promoting Sexual Health in Wales. Cardiff, UK: The National Assembly for Wales, 2000.

8 Botting B, Rosato M, Wood R. Teenage mothers and the health of their children. Popul Trends 1998; 93: 19-28.

9 Office for National Statistics - Social Survey Division. ONS Omnibus Survey, Contraception Module, January 1991 to November 2000 [Computer File]. Colchester, UK: UK Data Archive [Distributor], 2002

10 Dawe F, Meltzer H. Contraception and Sexual Health, 2000. London, UK: Office for National Statistics, 2002.

11 Department of Health. NHS Contraceptive Services, England: 2000-01. London, UK: Department of Health, 2001.

12 Office of Population Censuses and Surveys. Morbidity Statistics from General Practice. Fourth National Study 1991-1992. London, UK: HMSO, 1995.

13 Brook Advisory Centres. Brook Advisory Centres Annual Report 2000-01. London, UK: Brook Advisory Centres, 2001.

14 Rowlands S, Hannaford $P$. The incidence of sterilisation in the UK. Br J Obstet Gynaecol 2003; 110: 819-824.

15 National Centre for Social Research, Johnson AM, Fenton KA Copas A, McCadden A, Carder C, et al. National Survey of Sexual Attitudes and Lifestyles II, 2000-2001 [Computer File]. Colchester, UK: UK Data Archive [Distributor], 2005.

\section{APPENDIX}

\section{Calculation of relative change}

The relative change $(R C)$ is given by:

$\mathrm{RC}=100 \times[p(2000)-p(1991)] 10 \times p(1991)$

where $p(1991)$ and $p(2000)$ are fitted probabilities from a logistic regression of service use against year fitted to people within a subgroup. Estimation was performed using the 'svylogit' command in Stata. RC measures the average change per year between 1991 and 2000 as a percentage of the expected percentage using a service in 1991.

The fitted probabilities are obtained from the logistic regression of service use on year:

$\log [P(y) 1-P(y)]=\mathrm{A}+(y-1991) \times B$,

where $P(y)$ is the probability of having used a service in the last 5 years for someone in year $y=1991,1992$, etc., $A$ is the intercept term and $B$ is the regression slope. The $R C$ is based on a logistic rather than a normal linear regression model because the proportion using a service in some subgroups in some years is small, and could have resulted in negative predicted proportions.

Standard errors are obtained using the post-estimation commands in Stata. The estimated variances and covariance for parameters $A$ and $B$ are denoted by $v(A), v(B)$ and $c(A, B)$, respectively. The variance of $R C$ is given by the sandwich estimate:

$v(R C)=v(A) \times c(A, B) \times I^{2}+[v(A) v(B)+c(A, B)] \times I \times J+v(B) \times$ $c(A, B) \times \Omega$,

where $I=100 \times p(2000) \times\left\{1-[1-p(2000)]^{2}\right\}$ and $J=90 \times p(2000)$ $\times[1-p(2000)] p(1991)$ are the derivatives of $\mathrm{RC}$ with respect to $A$ and $B$, respectively. Thus, an approximate $95 \%$ confidence interval is given by:

$[\mathrm{RC}-1.96 \times \sqrt{ } v(\mathrm{RC}), \mathrm{RC}+1.96 \times \sqrt{ } v(\mathrm{RC})]$.

Note that $\mathrm{RC}$ depends on the regression coefficients such that $B$ $=0$ only when $R C=0$ (and vice versa). Thus, testing for trend by seeing if 0 lies outside the $\mathrm{Cl}$ is equivalent to testing $B=0$ and corresponds to a formal test of trend.
The successful bid will demonstrate:

- Appropriate leadership: the Director of the CEU is expected to be medically qualified, working in the speciality and a current Fellow, Member or Diplomate of the Faculty.

- A good knowledge and understanding of the provision of SRH care in the UK.

- Sustainable academic support.

- Sustainable administrative support.

- A clear mechanism for the delivery of each work stream outlined in the remit of the CEU above, ensuring continuity with the current work programme.

The Clinical Effectiveness Unit has an important role in supporting the members of the Faculty in their routine medical practice and promoting evidence-based medicine. There are three main ways in which the Unit will be expected to do this.

1. Developing new recommendations for clinical practice (evidence-based guidance products) and updating existing guidance when required.

2. Providing responses to Faculty members on clinical issues.

3. Review of new research or relevant pharmaceutical products.
- Cost effective use of resources.

An information pack is available from: Miss Jacquie Silcott, Faculty of Family Planning and Reproductive Health Care, 27 Sussex Place, Regent's Park, London NW1 4RG, UK.

Tel: 0207724 5536. E-mail: jsilcott@ffprhc.org.uk.

The closing date for applications is Monday 6th August 2007 Interviews will be held in early September 2007. 